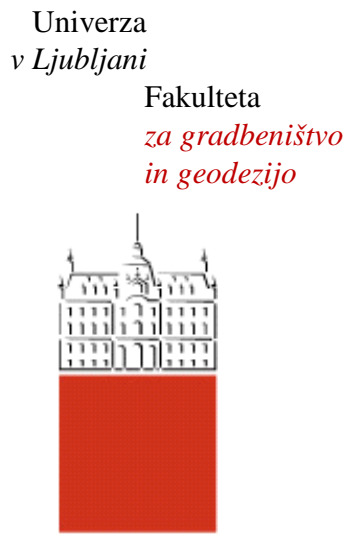

Jamova 2

1000 Ljubljana, Slovenija http://www3.fgg.uni-lj.si/

DRUGG - Digitalni repozitorij UL FGG http://drugg.fgg.uni-lj.si/

Ta članek je avtorjeva zadnja recenzirana različica, kot je bila sprejeta po opravljeni recenziji.

Prosimo, da se pri navajanju sklicujete na bibliografske podatke, kot je navedeno:
University

of Ljubljana

Faculty of

Civil and Geodetic

Engineering

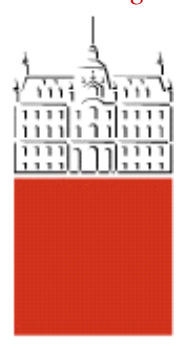

Jamova 2

SI - 1000 Ljubljana, Slovenia

http://www3.fgg.uni-lj.si/en/

DRUGG - The Digital Repository http://drugg.fgg.uni-lj.si/

This version of the article is author's manuscript as accepted for publishing after the review process.

When citing, please refer to the publisher's bibliographic information as follows:

Schnabl, S., Planinc, I., Saje, M., Čas, B. in Turk G. 2006. An analytical model of layered continuous beams with partial interaction. Structural engineering and mechanics 22, 3: 263278. 


\title{
An analytical model of layered continuous beams with partial interaction
}

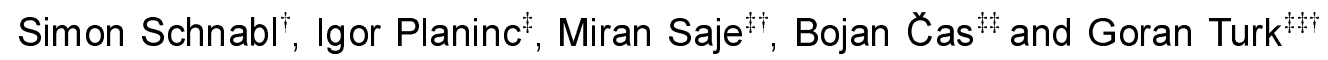 \\ University of Ljubljana, Faculty of Civil and Geodetic Engineering, Jamova 2, SI-1115 Ljubljana, Slovenia
}

(Received December 10, 2004, Accepted October 28, 2005)

\begin{abstract}
Starting with the geometrically non-linear formulation and the subsequent linearization, this paper presents a consistent formulation of the exact mechanical analysis of geometrically and materially linear three-layer continuous planar beams. Each layer of the beam is described by the geometrically linear beam theory. Constitutive laws of layer materials and relationships between interlayer slips and shear stresses at the interface are assumed to be linear elastic. The formulation is first applied in the analysis of a three-layer simply supported beam. The results are compared to those of Goodman and Popov (1968) and to those obtained from the formulation of the European code for timber structures, Eurocode 5 (1993). Comparisons show that the present and the Goodman and Popov (1968) results agree completely, while the Eurocode 5 (1993) results differ to a certain degree. Next, the analytical solution is used in formulating a general procedure for the analysis of layered continuous beams. The applications show the qualitative and quantitative effects of the layer and the interlayer slip stiffnesses on internal forces, stresses and deflections of composite continuous beams.
\end{abstract}

Key words: composite; layered beam; interlayer slip; mathematical model; analytical solution; elasticity.

\section{Introduction}

Due to their cost-effective construction and a good bearing capacity, layered composite systems are widely used in buildings and bridges. The behaviour of layered structures largely depends on the flexibility of a connection between the layers. Rigid connectors develop a full action between individual components, so that conventional principles of the solid beam analysis can be employed. Flexible connectors, on the other hand, permit the development of only partial interaction. As a result, an interlayer slip develops, with a sufficient magnitude to have a major effect on the deflection and stress distribution of the composite system.

When the force-slip relation of the connector is non-linear, which is usually the case, the response of the layered beam is also non-linear, even if component materials behave linearly. Consequently, the related non-linear mathematical model is described by the system of non-linear equations which

\footnotetext{
$\uparrow$ Young Researcher

+ Assistant Professor

t+ Professor, Corresponding author, E-mail: msaje@fgg.uni-lj.si

+t Research Associate

t+† Associate Professor
} 
can generally be solved only numerically. A variety of interesting formulations and numerical solution algorithms for the analysis of layered structures with continuous or discrete connectors has been proposed, e.g., Ayoub (2001), Ayoub and Filippou (2000), Čas et al. (2004a,b), Dall'Asta and Zona (2002), Fabbrocino et al. (2000), Faella et al. (2003), Fragiacomo et al. (2004), Gatessco (1999), Linden (1999), Oven et al. (1997), Thompson et al. (1975), Wheat and Calixto (1994), which consider, along with the non-linear tangential force-slip relation, also the non-linearity of material behaviour.

The building codes allow a structural engineer to perform a fully linear analysis for the determination of stress resultants. In a linear analysis, the governing equations of the mathematical model are linear and can be solved analytically. Many exact analytical solutions of simply supported, layered planar beams for combinations of simple loading cases and simple boundary conditions have been presented in professional literature, e.g., Adekola (1968), Cosenza and Pecce (2001), Fabbrocino et al. (2002), Girhammar and Gopu (1993), Girhammar and Pan (1993), Goodman and Popov (1968), Goodman and Popov (1969), Heinisuo (1988), Jasim (1997), Jasim and Mohamad (1997), Jasim (1999), Kristek and Studnicka (1982), Newmark et al. (1951), Nie et al. (2004), Ranzi and Bradford (2003), Silfwerbrand (1997), Smith and Teng (2001), Wang (1998). Some analytical solutions of two-layer continuous beams have also been presented (Jasim 1997, Plum and Horne 1975). Apart from the above presented numerical and analytical solutions a lot of experiments have been conducted on simply supported and continuous two-layer composite beams, e.g., Ansourian (1981), Newmark et al. (1951), Plum and Horne (1975), Wheat and Calixto (1994). On the other hand, there exist only few reports about experiments on simply supported three-layer composite beams (Goodman and Popov 1968, McCutheon 1986). To the author's best knowledge, there seems to be no exact solution reported on multilayered (three layers or more) simply supported and continuous beams having different material and geometric characteristics of layers. The present paper aims to fill the gap. Our formulation of the planar layered beam uses the following assumptions: (1) material is linear elastic; (2) displacements, strains and rotations are small; (3) shear deformations are disregarded (the 'Euler-Bernoulli beam'); (4) strains vary linearly over each layer (the 'Bernoulli hypothesis'); (5) the layers are continuously connected and the slip modulus of the connection is constant; (6) friction between the layers is not considered; (7) the bending strain is the same for all layers; (8) the number of layers is arbitrary; (9) the shapes of the cross-sections are symmetrical with respect to the deformation plane and preserve unchanged form and size during deformation.

For the purpose of clarity of presentation, our derivation is limited to the three-layer beam. The generalization to the multilayered beams is straightforward.

\section{Basic non-linear equations of a three-layer beam and their linearization}

Due to the exact geometrical introduction of interlayer slips between the layers, we started the derivation of our formulation with the non-linear kinematic and equilibrium equations first presented by Reissner (1972). After the linearization of Reissner's equations and taking into consideration the introduced assumptions, the simplified linear formulation of the three-layer beam with an interlayer slip is derived. Alternatively, the same linearized equilibrium equations can be obtained, if the linear kinematic equations are introduced in the principal of virtual displacements as constraining equations. However, in order to understand the actual kinematics, we belive it is important to start 
the derivation from the exact formulation. The present formulation assumes that the effect of shear strains is negligible. Our further assumption is that an interlayer tangential slip can occur at the interface between the layers, but no delamination or transverse separation between them is possible.

The mechanical behaviour of the composite beam is governed by the system of kinematic, equilibrium, and constitutive equations, combined with natural and essential boundary conditions for each layer, and with the constraining conditions for the contact between the layers.

\subsection{Kinematic, equilibrium and constitutive equations}

\subsubsection{Kinematic equations}

We consider an initially straight, planar, layered beam element of undeformed length $L$. Without a loss of generality, we assume that the layered beam element is made up of three layers. Layers are marked by letters $a, b$ and $c$ (see Fig. 1). The beam element is placed in the $(x, z)$-plane of a spatial Cartesian coordinate system with coordinates $(x, y, z)$ and unit base vectors $\mathbf{E}_{x}, \mathbf{E}_{y}, \mathbf{E}_{z}$. The undeformed reference axis of the layered beam element is common to all layers and is assumed to coincide with the geometric centroidal axis of the undeformed layered beam element. The layered beam element is subjected to conservative distributed loads $p_{x}, p_{z}$ and $m_{y}$ only along the span of layer $c$, and to generalized forces $S_{i}^{a}, S_{i}^{b}$ and $S_{i}^{c}(i=1,2, \ldots, 6)$ at the ends of layers $a, b$ and $c$.

The deformed configurations of layers $a, b$ and $c$ are defined by vector-valued functions

$$
\begin{gathered}
\mathbf{R}^{a}(x, z)=\left(x+u^{a}(x)+z \varphi^{a}(x)\right) \mathbf{E}_{x}+\left(z+w^{a}(x)\right) \mathbf{E}_{z} \\
\mathbf{R}^{b}\left(x^{*}, z\right)=\left(x^{*}+u^{b}\left(x^{*}\right)+z \varphi^{b}\left(x^{*}\right)\right) \mathbf{E}_{x}+\left(z+w^{b}\left(x^{*}\right)\right) \mathbf{E}_{z} \\
\mathbf{R}^{c}\left(x^{* *}, z\right)=\left(x^{* *}+u^{c}\left(x^{* *}\right)+z \varphi^{c}\left(x^{* *}\right)\right) \mathbf{E}_{x}+\left(z+w^{c}\left(x^{* *}\right)\right) \mathbf{E}_{z}
\end{gathered}
$$

In Eqs. (1) and in all further expressions, the notations $(\bullet)^{a},(\bullet)^{b}$ and $(\bullet)^{c}$ mark that quanatities $(\bullet)$ are related to layer $a, b$ or $c$. Functions $u^{a}, w^{a}, \varphi^{a}$ denote the components of the displacement vector and the rotation angle of layer $a$ at the reference axis with respect to the base vectors $\mathbf{E}_{x}, \mathbf{E}_{z}$ and $\mathbf{E}_{y}$, respectively. Variables $u^{b}, w^{b}, \varphi^{b}, u^{c}, w^{c}, \varphi^{c}$ are related to layers $b$ and $c$.

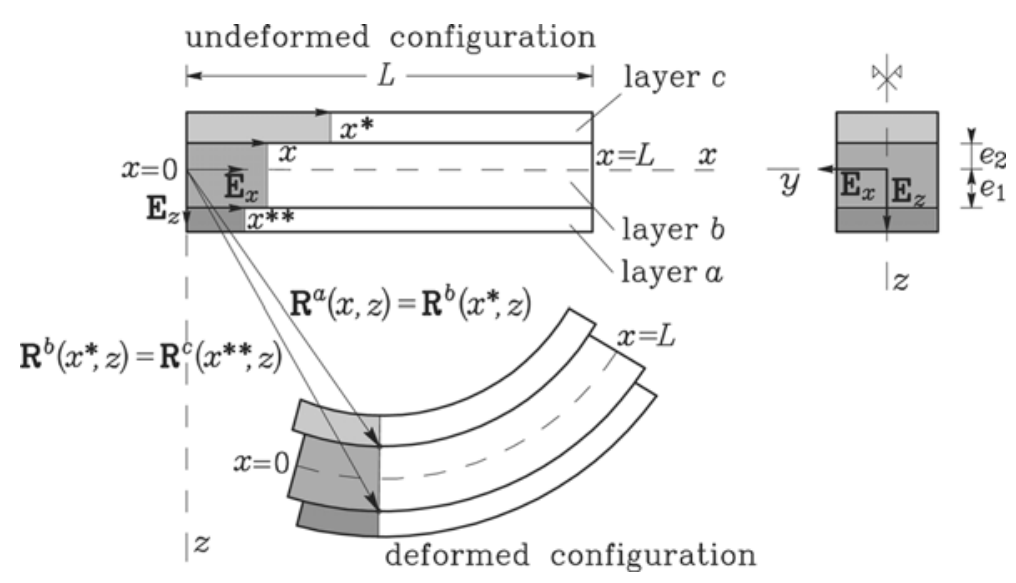

Fig. 1 Undeformed and deformed configuration of the three-layer beam 
If the effect of shear strains is neglected, Reissner's equations read (Reissner 1972)

$$
\begin{gathered}
1+u^{\prime}-(1+\varepsilon) \cos \varphi=0 \\
w^{\prime}+(1+\varepsilon) \sin \varphi=0 \\
\varphi^{\prime}-\kappa=0
\end{gathered}
$$

In Eqs. (2) the prime (') denotes the derivative with respect to $x$, whereas the functions $\varepsilon, \varphi$ and $\kappa$ mark the extensional strain (or the specific elongation), the rotation and the bending strain of the reference axis, respectively.

The linearized kinematic equations are obtained by the linearization of Eqs. (2) around the undeformed configuration for each layer. After the linearization has been completed, we obtain

$$
\begin{array}{lll}
\delta u^{a \prime}-\delta \varepsilon^{a}=0 & \delta u^{b^{\prime}}-\delta \varepsilon^{b}=0 & \delta u^{c \prime}-\delta \varepsilon^{c}=0 \\
\delta w^{a \prime}+\delta \varphi^{a}=0 & \delta w^{b^{\prime}}+\delta \varphi^{b}=0 & \delta w^{c \prime}+\delta \varphi^{c}=0 \\
\delta \varphi^{a \prime}-\delta \kappa^{a}=0 & \delta \varphi^{b \prime}-\delta \kappa^{b}=0 & \delta \varphi^{c \prime}-\delta \kappa^{c}=0
\end{array}
$$

Here, $\delta u^{a \prime}, \delta \varepsilon^{a}, \ldots, \delta \kappa^{c}$ are the variations of the independent variables. As the independent variables $\left[u^{a \prime}, \varepsilon^{a}, \ldots, \kappa^{c}\right]$ are zero in the undeformed configuration, we can without any loss of clarity of the presentation change the notations and drop the variation sign ' $\delta$ '. Eqs. (3) will then assume the form

$$
\begin{array}{lll}
u^{a \prime}-\varepsilon^{a}=0 & u^{b \prime}-\varepsilon^{b}=0 & u^{c \prime}-\varepsilon^{c}=0 \\
w^{a \prime}+\varphi^{a}=0 & w^{b \prime}+\varphi^{b}=0 & w^{c \prime}+\varphi^{c}=0 \\
\varphi^{a \prime}-\kappa^{a}=0 & \varphi^{b \prime}-\kappa^{b}=0 & \varphi^{c \prime}-\kappa^{c}=0
\end{array}
$$

$u^{a}, u^{b}, u^{c}, w^{a}, w^{b}, w^{c}, \varepsilon^{a}, \varepsilon^{b}, \varepsilon^{c}, \kappa^{a}, \kappa^{b}, \kappa^{c}, \varphi^{a}, \varphi^{b}, \varphi^{c}$ now play the role of displacements, strains and rotations of the linearized theory. These variables are assumed to be small.

The geometric or total extensional strains, $D^{a}, D^{b}$ and $D^{c}$, of an arbitrary fibre in layers $a, b$ and $c$ are functions of extensional strains $\varepsilon^{a}, \varepsilon^{b}$ and $\varepsilon^{c}$ and bending strains $\kappa^{a}, \kappa^{b}$ and $\kappa^{c}$ of the reference axes of the layers. According to Bernoulli's hypothesis, they are linear functions of $z$

$$
\begin{aligned}
& D^{a}(x, z)=\varepsilon^{a}(x)+z \kappa^{a}(x) \\
& D^{b}(x, z)=\varepsilon^{b}(x)+z \kappa^{b}(x) \\
& D^{c}(x, z)=\varepsilon^{c}(x)+z \kappa^{c}(x)
\end{aligned}
$$

The substitution of $\varepsilon^{a}, \varepsilon^{b}, \varepsilon^{c}$ with $u^{a}, u^{b}, u^{c}$ using Eqs. (4) gives

$$
\begin{aligned}
& u^{a \prime}(x)-D^{a}(x, z)+z \kappa^{a}(x)=0 \\
& u^{b \prime}(x)-D^{b}(x, z)+z \kappa^{b}(x)=0 \\
& u^{c \prime}(x)-D^{c}(x, z)+z \kappa^{c}(x)=0
\end{aligned}
$$




\subsubsection{Equilibrium equations}

When in equilibrium, the axial force $\mathcal{N}$, the shear force $\mathcal{Q}$, the bending moment $\mathcal{M}$, and the distributed loads $p_{x}, p_{z}$ and $m_{y}$, must satisfy the equilibrium equations (Reissner 1972)

$$
\begin{gathered}
\mathcal{N}^{\prime}+p_{x}=0 \\
\mathcal{Q}^{\prime}+p_{z}=0 \\
\mathcal{M}^{\prime}-(1+\varepsilon) \mathcal{Q}+m_{y}=0
\end{gathered}
$$

Once the equilibrium equations have been written for all three layers and linearized, they read

$$
\begin{aligned}
\mathcal{N}^{a \prime}-p_{t}^{a b}=0 & \mathcal{N}^{b \prime}+p_{t}^{a b}-p_{t}^{b c}=0 & \mathcal{N}^{c \prime}+p_{x}+p_{t}^{b c}=0 \\
\mathcal{Q}^{a \prime}+p_{n}^{a b}=0 & \mathcal{Q}^{b \prime}-p_{n}^{a b}+p_{n}^{b c}=0 & \mathcal{Q}^{c \prime}-p_{n}^{b c}+p_{z}=0 \\
\mathcal{M}^{a \prime}-\mathcal{Q}^{a}-p_{t}^{a b} e_{1}=0 & \mathcal{M}^{b \prime}-\mathcal{Q}^{b}+p_{t}^{a b} e_{1}+p_{t}^{b c} e_{2}=0 & \mathcal{M}^{c \prime}-\mathcal{Q}^{c}-p_{t}^{b c} e_{2}=0
\end{aligned}
$$

Here $p_{t}^{a b}, p_{t}^{b c}$ and $p_{n}^{a b}, p_{n}^{b c}$ represent the tangential and normal interlayer contact tractions. The distances from the reference axis to the contact planes between the layers are denoted by $e_{1}$ and $e_{2}$ (Fig. 1).

\subsubsection{Constitutive equations}

The third set of the basic equations is provided by the constitutive law. The constitutive law relates the stress resultants, i.e., the generalized forces $\mathcal{N}_{C}^{a}, \mathcal{N}_{C}^{b}, \mathcal{N}_{C}^{c}, \mathcal{M}_{C}^{a}, \mathcal{M}_{C}^{b}, \mathcal{M}_{C}^{c}$ to the equilibrium internal forces, $\mathcal{N}^{a}, \mathcal{N}^{b}, \mathcal{N}^{c}, \mathcal{M}^{a}, \mathcal{M}^{b}, \mathcal{M}^{c}$, which are the solutions of Eqs. (8). These relations assume the form

$$
\begin{array}{ll}
\mathcal{N}^{a}-\mathcal{N}_{C}^{a}\left(\varepsilon^{a}, \kappa^{a}\right)=0 & \mathcal{M}^{a}-\mathcal{M}_{C}^{a}\left(\varepsilon^{a}, \kappa^{a}\right)=0 \\
\mathcal{N}^{b}-\mathcal{N}_{C}^{b}\left(\varepsilon^{b}, \kappa^{b}\right)=0 & \mathcal{M}^{b}-\mathcal{M}_{C}^{b}\left(\varepsilon^{b}, \kappa^{b}\right)=0 \\
\mathcal{N}^{c}-\mathcal{N}_{C}^{c}\left(\varepsilon^{c}, \kappa^{c}\right)=0 & \mathcal{M}^{c}-\mathcal{M}_{C}^{c}\left(\varepsilon^{c}, \kappa^{c}\right)=0
\end{array}
$$

We postulate that layer material is linear elastic. Therefore, Eqs. (9) are assumed to be given by the linear relations with respect to $\varepsilon$ and $\kappa$.

$$
\begin{array}{ll}
\mathcal{N}_{C}^{a}=C_{11}^{a} \varepsilon^{a}+C_{12}^{a} \kappa^{a} & \mathcal{M}_{C}^{a}=C_{21}^{a} \varepsilon^{a}+C_{22}^{a} \kappa^{a} \\
\mathcal{N}_{C}^{b}=C_{11}^{b} \varepsilon^{b}+C_{12}^{b} \kappa^{b} & \mathcal{M}_{C}^{b}=C_{21}^{b} \varepsilon^{b}+C_{22}^{b} \kappa^{b} \\
\mathcal{N}_{C}^{c}=C_{11}^{c} \varepsilon^{c}+C_{12}^{c} \kappa^{c} & \mathcal{M}_{C}^{c}=C_{21}^{c} \varepsilon^{c}+C_{22}^{c} \kappa^{c}
\end{array}
$$

in which material and geometrical constants are marked by $C_{11}^{a}, C_{12}^{a}, \ldots, C_{22}^{c}$; e.g., $C_{22}^{a}=E_{a} J_{a}$, where $E_{a}$ is elastic modulus of layer $a$, and $J_{a}$ is the moment of inertia of layer $a$ with respect to the geometric centroidal point of the whole cross-section. 


\subsection{Constraining equations}

The constraining equations define the conditions that assemble an individual layer into a layered composite beam.

As already stated, the transverse separation or penetration between the layers is not allowed. This fact is expressed by the requirements

$$
\begin{aligned}
\mathbf{R}^{a}(x, z) & =\mathbf{R}^{b}\left(x^{*}, z\right) \\
\mathbf{R}^{b}\left(x^{*}, z\right) & =\mathbf{R}^{c}\left(x^{* *}, z\right)
\end{aligned}
$$

where $x \in \mathcal{I}^{a}, x^{*} \in \mathcal{I}^{b}$ are undeformed coordinates of two distinct particles of layers $a$ and $b$ which are in the deformed state in contact, and thus their vector-valued functions $\mathbf{R}^{a}(x, z)$ and $\mathbf{R}^{b}\left(x^{*}, z\right)$ coincide. Similarly, $x^{* *} \in \mathcal{I}^{c}$ is a material, undeformed coordinate of particle of layer $c$, which is in the deformed state in contact with particle of layer $b$, and therefore their vector-valued functions $\mathbf{R}^{b}\left(x^{*}, z\right)$ and $\mathbf{R}^{c}\left(x^{* *}, z\right)$ in the deformed state coincide (see Fig. 1). The contact regions of layers $a$, $b$ and $c$ are marked by $\mathcal{I}^{a}, \mathcal{I}^{b}, \mathcal{I}^{c}$. Eqs. (11) are rewritten in a more convenient component form:

$$
\begin{aligned}
x+u^{a}(x) & =x^{*}+u^{b}\left(x^{*}\right) \\
x^{*}+u^{b}\left(x^{*}\right) & =x^{* *}+u^{c}\left(x^{* *}\right) \\
w^{a}(x) & =w^{b}\left(x^{*}\right) \\
w^{b}\left(x^{*}\right) & =w^{c}\left(x^{* *}\right)
\end{aligned}
$$

The relative tangential displacement (slip) that occurs between the two particles which coincide in the undeformed configuration is denoted by $\Delta$. By definition, it is the difference of the deformed arc-lengths of contact curves of layers $a$ and $b$, and $b$ and $c$. For a geometrically linear layered beam theory, the arc-length differences can easily be calculated and are simply given by

$$
\Delta^{a b}=U^{a}-U^{b} \quad \Delta^{b c}=U^{b}-U^{c}
$$

$U^{a}, U^{b}, U^{c}$ denote displacements of a particular material particle of the related layer at the interlayer contact.

In general, the flexibility of the contact highly depends on the characteristics of layer materials and on the way the contact is enforced. The constitutive law of the bond slip between the layers generally assumes the form

$$
p_{t}^{a b}=\mathcal{F}^{a b}\left(\Delta^{a b}\right) \quad p_{t}^{b c}=\mathcal{F}^{b c}\left(\Delta^{b c}\right)
$$

where $\mathcal{F}^{a b}$ and $\mathcal{F}^{b c}$ can be arbitrary non-linear functions. In the present paper, however, we will assume the linear relationships between $p_{t}$ and $\Delta$, see Eqs. (30), as it is generally proposed by structural engineering standards (e.g., Eurocode 5(1993)). In this case the analytical solution of the problem can be derived. On the other hand, if the problem is solved numerically, a general nonlinear relationship in Eqs. (14) can be assumed. 


\subsection{System of equations of a three-layer beam with an interlayer slip}

Since deformations, displacements and rotations are assumed to be small quantities, the generalized equilibrium equations can further be simplified using the following two assumptions (see, e.g., Čas et al. 2004b): (i) $d x \cong d x^{*} \cong d x^{* *}$; (ii) bending strains of the reference axis of individual layers are equal $\kappa^{a}(x)=\kappa^{b}\left(x^{*}\right)=\kappa^{c}\left(x^{* *}\right)=\kappa(x)$. Consequently, $\varphi^{a}(x)=\varphi^{b}\left(x^{*}\right)$ $=\varphi^{c}\left(x^{* *}\right)=\varphi(x), w^{a}(x)=w^{b}\left(x^{*}\right)=w^{c}\left(x^{* *}\right)=w(x), \mathcal{I}^{a} \cong \mathcal{I}^{b} \cong \mathcal{I}^{c}=[0, L]$. Thus, $(\bullet)^{b}\left(x^{*}\right)=(\bullet)^{b}(x)$, $(\bullet)^{c}\left(x^{* *}\right)=(\bullet)^{c}(x)$ holds true for any quantity of layers $b$ and $c$, e.g., $u^{c}\left(x^{* *}\right)=u^{c}(x)$. Kinematic, equilibrium and constraining equations can now be considerably simplified. After considering the assumptions mentioned above, we can decompose the basic equations of the three-layer beam with an interlayer slip into two separate systems of differential and algebraic equations (see, e.g., Čas et al. 2004b):

$$
\begin{gathered}
u^{a \prime}-\varepsilon^{a}=0 \\
u^{b^{\prime}}-\varepsilon^{b}=0 \\
u^{c \prime}-\varepsilon^{c}=0 \\
w^{\prime}+\varphi=0 \\
\varphi^{\prime}-\kappa=0 \\
\mathcal{N}^{a \prime}-p_{t}^{a b}=0 \\
\mathcal{N}^{b \prime}+p_{t}^{a b}-p_{t}^{b c}=0 \\
\mathcal{N}^{c \prime}+p_{t}^{b c}+p_{x}=0 \\
\left(\mathcal{Q}^{a}+\mathcal{Q}^{b}+\mathcal{Q}^{c}\right)^{\prime}+p_{z}=0 \rightarrow \mathcal{Q}^{\prime}+p_{z}=0 \\
\left.\mathcal{M}^{c}\right)^{\prime}-\left(\mathcal{Q}^{b}+\mathcal{Q}^{c}\right)+m_{y}=0 \rightarrow \mathcal{M}^{\prime}-\mathcal{Q}+m_{y}=0 \\
\mathcal{N}^{a}=\mathcal{N}_{C}^{a}=C_{11}^{a} \varepsilon^{a}+C_{12}^{a} \kappa \\
\mathcal{N}^{b}=\mathcal{N}_{C}^{b}=C_{11}^{b} \varepsilon^{b}+C_{12}^{b} \kappa \\
\mathcal{N}^{c}=\mathcal{N}_{C}^{c}=C_{11}^{c} \varepsilon^{c}+C_{12}^{c} \kappa \\
\mathcal{M}=\mathcal{M}_{C}^{a}+\mathcal{M}_{C}^{b}+\mathcal{M}_{C}^{c} \rightarrow \mathcal{M}^{b}=C_{21}^{a} \varepsilon^{a}+C_{21}^{b} \varepsilon^{b}+C_{21}^{c} \varepsilon^{c}+\left(C_{22}^{a}+C_{22}^{b}+C_{22}^{c}\right) \kappa \\
\Delta^{a b}=u^{a}-u^{b} \\
\Delta^{b c}=u^{b}-u^{c}
\end{gathered}
$$




$$
\begin{aligned}
& p_{t}^{a b}=\mathcal{F}^{a b}\left(\Delta^{a b}\right) \rightarrow p_{t}^{a b}=K^{a b} \Delta^{a b} \\
& p_{t}^{b c}=\mathcal{F}^{b c}\left(\Delta^{b c}\right) \rightarrow p_{t}^{b c}=K^{b c} \Delta^{b c}
\end{aligned}
$$

and

$$
\begin{gathered}
x+u^{a}=x^{*}+u^{b} \rightarrow x^{*}=x+\Delta^{a b} \\
x^{*}+u^{b}=x^{* *}+u^{c} \rightarrow x^{* *}=x^{*}+\Delta^{b c} \\
\mathcal{Q}^{a \prime}+p_{n}^{a b}=0, \mathcal{Q}^{b \prime}-p_{n}^{a b}+p_{n}^{b c}=0 \\
\mathcal{M}^{a \prime}-\mathcal{Q}^{a}=0, \mathcal{M}^{b \prime}-\mathcal{Q}^{b}=0 \\
\mathcal{M}^{a}=\mathcal{M}_{C}^{a}=C_{21}^{a} \varepsilon^{a}+C_{22}^{a} \kappa \text { and } \mathcal{M}^{b}=\mathcal{M}_{C}^{b}=C_{21}^{b} \varepsilon^{b}+C_{22}^{b} \kappa \\
\mathcal{Q}=\mathcal{Q}^{a}+\mathcal{Q}^{b}+\mathcal{Q}^{c} \\
\mathcal{M}=\mathcal{M}^{a}+\mathcal{M}^{b}+\mathcal{M}^{c}
\end{gathered}
$$

Eqs. (15)-(30) consist of 18 equations for 18 unknown functions $u^{a}, u^{b}, u^{c}, w, \varphi, \varepsilon^{a}, \varepsilon^{b}, \varepsilon^{c}, \kappa, \mathcal{N}^{a}$, $\mathcal{N}^{b}, \mathcal{N}^{c}, \mathcal{Q}, \mathcal{M}, \Delta^{a b}, \Delta^{b c}, p_{t}^{a b}, p_{t}^{b c}$, whereas Eqs. (31)-(37) constitute a system of ten equations for ten unknown functions $x^{*}, x^{* *}, p_{n}^{a b}, p_{n}^{b c}, \mathcal{Q}^{a}, \mathcal{Q}^{b}, \mathcal{Q}^{c}, \mathcal{M}^{a}, \mathcal{M}^{b}, \mathcal{M}^{c}$. In Eqs. (30), $K^{a b}$ and $K^{b c}$ denote the slip moduli of connections.

\section{Solution algorithm}

If the slips between the layers are known functions of $x$, the solution of the system of Eqs. (15)(37) can easily be obtained with the following sequence of tasks.

In the first step, we integrate Eqs. (23) and (24) and obtain the expressions for the total equilibrium shear force and the total equilibrium bending moment of the three-layer beam element:

$$
\begin{gathered}
\mathcal{Q}(x)=\mathcal{Q}(0)-\int_{0}^{x} p_{z}(\xi) d \xi \\
\mathcal{M}(x)=\mathcal{M}(0)+\int_{0}^{x}\left(\mathcal{Q}(\xi)-m_{y}(\xi)\right) d \xi
\end{gathered}
$$

In the second step, we twice differentiate Eqs. (29) with respect to $x$ and insert Eqs. (15)-(16). The following differential equations for the interlayer slips emerge

$$
\begin{aligned}
& \Delta^{a b \prime \prime}=\varepsilon^{a \prime}-\varepsilon^{b^{\prime}} \\
& \Delta^{b c \prime \prime}=\varepsilon^{b^{\prime}}-\varepsilon^{c^{\prime}}
\end{aligned}
$$

The derivatives $\varepsilon^{a \prime}, \varepsilon^{b \prime}$ and $\varepsilon^{c \prime}$ are obtained by the differentiation of Eqs. (25)-(28) with respect to 
$x$. Solving the differentiated Eqs. (25)-(28) for $\varepsilon^{a \prime}, \varepsilon^{b \prime}, \varepsilon^{c \prime}$ and $\kappa^{\prime}$ yields

$$
\left\{\begin{array}{l}
\varepsilon^{a \prime} \\
\varepsilon^{b \prime} \\
\varepsilon^{c \prime} \\
\kappa^{\prime}
\end{array}\right\}=\mathbf{C}^{-1}\left\{\begin{array}{l}
\mathcal{N}^{a \prime} \\
\mathcal{N}^{b \prime} \\
\mathcal{N}^{c \prime} \\
\mathcal{M}^{\prime}
\end{array}\right\}
$$

$\mathbf{C}$ is the matrix of constitutive constants (see Eqs. (10)), and $\mathbf{C}^{-1}$ is its inverse:

$$
\mathbf{C}^{-1}=\left[\begin{array}{cccc}
C_{11}^{a} & 0 & 0 & C_{12}^{a} \\
0 & C_{11}^{b} & 0 & C_{12}^{b} \\
0 & 0 & C_{11}^{c} & C_{12}^{c} \\
C_{21}^{a} & C_{21}^{b} & C_{21}^{c} & C_{22}^{a}+C_{22}^{b}+C_{22}^{c}
\end{array}\right]^{-1}=\left[\begin{array}{cccc}
D_{11} & 0 & 0 & D_{14} \\
0 & D_{22} & 0 & D_{24} \\
0 & 0 & D_{33} & D_{34} \\
D_{41} & D_{42} & D_{43} & D_{44}
\end{array}\right]
$$

When Eqs. (20)-(24), (30) and (40) are inserted into Eqs. (39), we obtain a system of two secondorder linear differential equations with constant coefficients for the slips between layers $a, b$ and $c$

$$
\begin{aligned}
& \Delta^{a b \prime \prime}-A^{a b} \Delta^{a b}+B^{a b} \Delta^{b c}=f^{a b} \\
& \Delta^{b c \prime \prime}+A^{b c} \Delta^{a b}-B^{b c} \Delta^{b c}=f^{b c}
\end{aligned}
$$

with $A^{a b}, B^{a b}, A^{b c}, B^{b c}$ and $f^{a b}, f^{b c}$ being constants

$$
\begin{gathered}
A^{a b}=K^{a b}\left(D_{11}+D_{22}\right) \quad B^{a b}=K^{b c} D_{22} \\
A^{b c}=K^{a b} D_{22} \quad B^{b c}=K^{b c}\left(D_{22}-D_{33}\right) \\
f^{a b}=\left(D_{14}-D_{24}\right)\left(\mathcal{Q}-m_{y}\right) \\
f^{b c}=D_{22} p_{x}+\left(D_{34}-D_{24}\right)\left(\mathcal{Q}-m_{y}\right)
\end{gathered}
$$

Boundary conditions associated with Eqs. (42) are the values of the interlayer slips at the edges $x=0$ and $x=L$ of the beam element. An exact solution of Eqs. (42) was obtained by MATHEMATICA (2003). After the slips have been obtained, the remaining equations of the system (15)-(30) can simply be solved. We first determine the boundary rotations and displacements from the system of linear equations

$$
\mathbf{K}_{T} \mathbf{u}=\mathbf{g}
$$

for the composite structure. In Eq. (45), $\mathbf{K}_{T}$ denotes the tangent stiffness matrix, $\mathbf{u}$ is the vector of end-point displacements, and $\mathbf{g}$ is the load vector. Once $\mathbf{u}$ has been known, the values of the end forces can easily be computed. This step completes the solution of the system (15)-(30) for unknown functions $u^{a}, u^{b}, u^{c}, w, \varphi, \varepsilon^{a}, \varepsilon^{b}, \varepsilon^{c}, \kappa, \mathcal{N}^{a}, \mathcal{N}^{b}, \mathcal{N}^{c}, \mathcal{Q}, \mathcal{M}, \Delta^{a b}, \Delta^{b c}, p_{t}^{a b}, p_{t}^{b c}$. Finally, the unknown functions $x^{*}, x^{* *}, p_{n}^{a b}, p_{n}^{b c}, \mathcal{Q}^{a}, \mathcal{Q}^{b}, \mathcal{Q}^{c}, \mathcal{M}^{a}, \mathcal{M}^{b}, \mathcal{M}^{c}$ are obtained from Eqs. (31)-(37). 
The interlayer tractions in the normal direction of the contact are obtained from equations

$$
\begin{gathered}
p_{n}^{a b}=-\mathcal{M}_{C}^{a \prime \prime}-p_{t}^{a b \prime} e_{1} \\
p_{n}^{b c}=-\mathcal{M}_{C}^{b \prime \prime}-p_{t}^{a b \prime} e_{1}-p_{t}^{b c \prime} e_{2}+p_{n}^{a b}
\end{gathered}
$$

which are derived if we insert Eqs. (33) into Eqs. (34).

\section{Numerical examples}

Numerical examples will demonstrate the ability of the present exact analytical model for the stress-strain analysis of the three-layer simply supported and continuous beams with partial interaction between the layers the geometric and material characteristics of which may vary over the cross-section. They will describe accurately the behaviour of layered beams at small elastic strains and will also provide for the referential, or bench-mark solutions suitable for assessing the accuracy of new finite element formulations. We consider two cases: (i) a simply supported three-layer beam; and (ii) a continuous three-layer beam over two spans.

\subsection{A simply supported three-layer beam}

The geometrically and materially linear models of two-layer beams are often encountered in literature (see, e.g., Adekola 1968, Cosenza and Peece 2001, Čas et al. 2004b, Girhammar and Gopu 1993, Girhammar and Pan 1993, Goodman and Popov 1968, 1969, Jasim 1997, 1999, Jasim and Mohamad 1997, Kristek and Studnicka 1982, Newmark et al. 1951, Plum and Horne 1975, Ranzi and Bradford 2003, Rassam and Goodman 1971, Smith and Teng 2001).

In contrast, the analytical solution for the three-layer simply supported composite beam appears only to be derived by Goodman and Popov (1968). Moreover, some experiments have been conducted on these types of beams. They analysed a simply supported three-layer beam subjected to force $P$ at the mid-span of the timber beam, with layers of equal thickness and made up of the same wood material. Here, we analyse this simply supported three-layer beam subjected both to point load and uniformly distributed load. The descriptive geometric, material and loading data are given in Fig. 2.

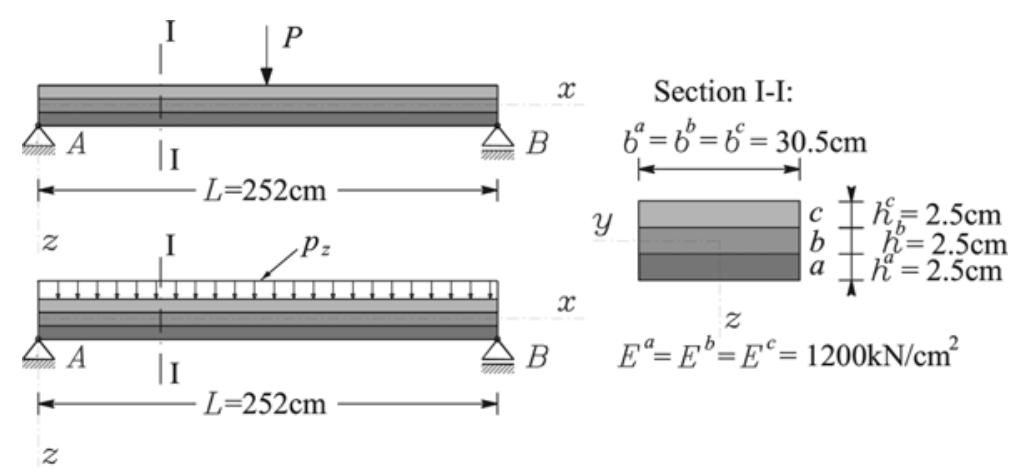

Fig. 2 Simply supported three-layer beam 
The contributions of the connection flexibility to the vertical deflections at the mid-span have been calculated for various values of the slip moduli of connections and are shown in Tables 1 and 2 for the comparison reasons in the numerical form rather than graphically. The tables also present the comparisons with the results in Goodman and Popov (1968), and with the results given by Eurocode 5 formulae (1993). The results are presented for the range of the slip modulus from 0.01 to $100 \mathrm{kN} / \mathrm{cm}^{2}$. Observe that the results of Goodman and Popov (1968) and of the present formulation are in perfect agreement both for the point and the distributed load. Since, the validation of Goodman and Popov mathematical model shows excellent agreement with all their

Table 1 Simply supported three-layer beam subjected to the point load. The contribution of the flexible connection to the vertical deflections in cm as a function of slip modulus, $K=K^{a b}=K^{b c}$. Deflection of a solid beam is $0.246 \mathrm{~cm}$.

\begin{tabular}{|c|c|c|c|c|}
\hline \multicolumn{5}{|c|}{$P=1 \mathrm{kN}$} \\
\hline$K\left[\mathrm{kN} / \mathrm{cm}^{2}\right]$ & Goodman (1968) & Present & EC 5 (1993) & $\begin{array}{l}\text { EC } 5(1993) \\
\text { relative error [\%] }\end{array}$ \\
\hline 0.01 & 1.953 & 1.953 & 1.953 & -0.01 \\
\hline 0.1 & 1.852 & 1.852 & 1.852 & -0.08 \\
\hline 0.5 & 1.506 & 1.506 & 1.499 & 0.39 \\
\hline 1 & 1.222 & 1.222 & 1.212 & 0.76 \\
\hline 2 & 0.899 & 0.899 & 0.877 & 1.45 \\
\hline 3 & 0.701 & 0.701 & 0.686 & 2.06 \\
\hline 4 & 0.579 & 0.579 & 0.564 & 2.62 \\
\hline 5 & 0.494 & 0.494 & 0.479 & 3.12 \\
\hline 10 & 0.287 & 0.287 & 0.273 & 5.13 \\
\hline 100 & 0.035 & 0.035 & 0.031 & 13.56 \\
\hline
\end{tabular}

Table 2 Simply supported three-layer beam subjected to the uniform load. The contribution of the flexible connection to the vertical deflections in $\mathrm{cm}$ as a function of slip modulus, $K=K^{a b}=K^{b c}$. Deflection of a solid beam is $0.386 \mathrm{~cm}$.

\begin{tabular}{ccccc}
\hline \hline \multicolumn{5}{c}{$p_{z}=1 \mathrm{kN} / \mathrm{m}$} \\
\hline$K\left[\mathrm{kN} / \mathrm{cm}^{2}\right]$ & Goodman (1968) & Present & EC 5 (1993) & $\begin{array}{c}\text { EC 5 (1993) } \\
\text { relative error [\%] }\end{array}$ \\
\hline 0.01 & 3.069 & 3.069 & 3.069 & 0.00 \\
0.1 & 2.907 & 2.907 & 2.908 & 0.02 \\
0.5 & 2.355 & 2.355 & 2.357 & 0.10 \\
1 & 1.902 & 1.902 & 1.906 & 0.20 \\
2 & 1.373 & 1.373 & 1.378 & 0.37 \\
3 & 1.073 & 1.073 & 1.079 & 0.52 \\
4 & 0.881 & 0.881 & 0.887 & 0.66 \\
5 & 0.747 & 0.747 & 0.753 & 0.78 \\
10 & 0.423 & 0.423 & 0.429 & 1.24 \\
100 & 0.048 & 0.048 & 0.049 & 2.52 \\
\hline
\end{tabular}




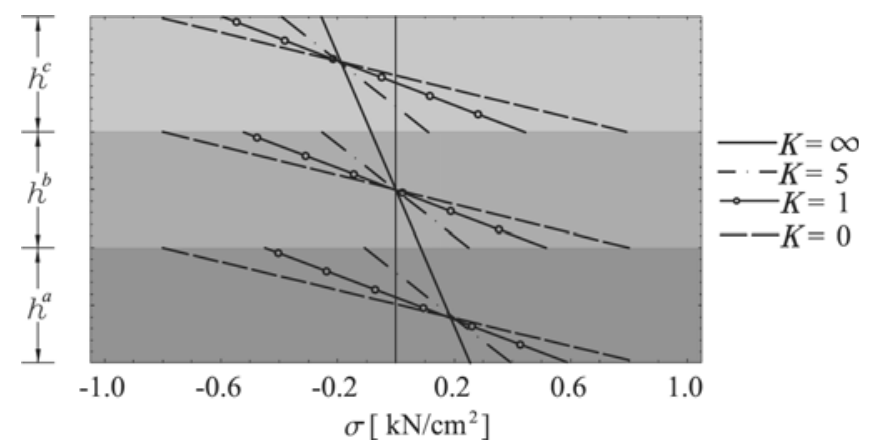

Fig. 3 Simply supported three-layer beam subjected to point load. The distribution of the normal stresses over the cross-section.

experimental results, we can conclude that the present formulation is in good correlation with the experiments performed on three-layer beams subjected to serviceability loads.

When the beam is subjected to the point load, the analytical values for the contributions to the vertical deflections at small values of interlayer slip modulus $\left(K<1 \mathrm{kN} / \mathrm{m}^{2}\right)$ are almost equal to those in Eurocode 5 (1993), although Eurocode 5 limits its application to the distributed load. For higher values of slip moduli, they are somewhat smaller. Thus, we see that Eurocode 5 (1993) gives rather accurate deflections (although somewhat smaller) even in the case of a point load.

When the beam is subjected to the uniformly distributed load, the Eurocode 5 (1993) results practically agree with the analytical results for any $K$.

The values in Table 2 indicate that the contributions to the deflections due to the nonstiff interaction between the layers may be as big as about 8 -times the deflections in rigidly connected layered beam, which is equal to $0.386 \mathrm{~cm}$.

One of the basic assumptions of the Goodman and Popov (1968) model is that the thicknesses of layers are equal, which leads to the assumption that the bending moments and the axial forces are equal in each layer. This assumption holds, provided that the layers have identical material and geometric characteristics. However, it may cause large errors in results, if this criterion is not fulfilled, as can be seen in the example below.

The distributions and the values of the normal stresses in the layers are very much affected by the degree of the contact rigidity. The effect is depicted in Fig. 3 for stiffnesses $K=0,1,5$ and $\infty$. The stress distributions over the mid-point section of the beam subjected to the point load are shown. We can see that the peak values of the stress in each layer increase with the decreasing stiffness of the contact, the values with regard to the absolutely stiff contact being substantially larger. Note that for the flexible contact, the stresses in each layer vary from tension on the bottom side of the layer to compression on its top (the 'zig zag' variation).

\subsection{A continuous three-layer timber beam over two spans}

We study a continuous three-layer beam the layers of which are made up of timber of strength classes defined in Eurocode 5 (1993). In addition, slip moduli between the layers are different: $K^{a b}=$ $3 \mathrm{kN} / \mathrm{cm}^{2}$ and $K^{b c}=0.01 \mathrm{kN} / \mathrm{cm}^{2}$. The continuous beam is subjected to the uniformly distributed load $p_{z}=0.01 \mathrm{kN} / \mathrm{cm}$. The geometric, material and loading data are given in Fig. 4. 


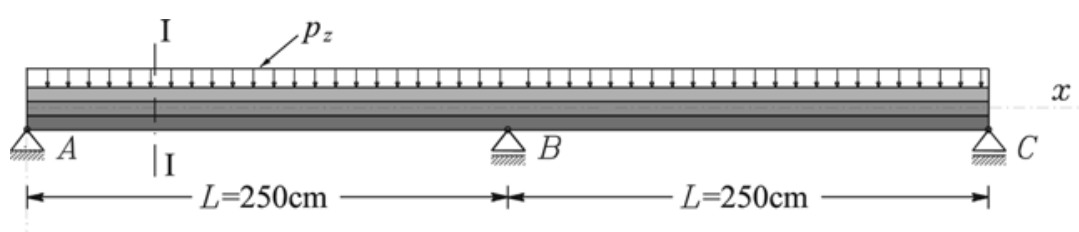

$z$

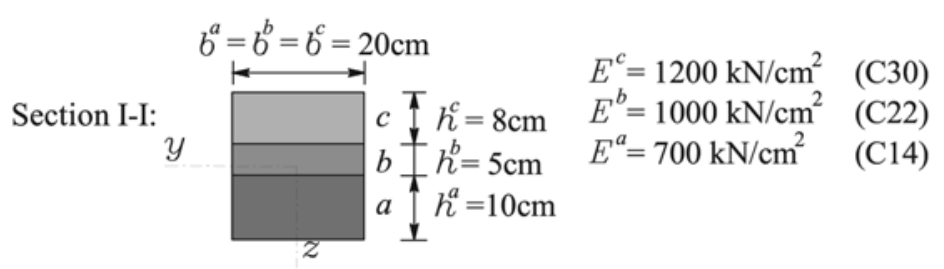

Fig. 4 Continuous three-layer beam over two spans

Table 3 Continuous beam over two spans (Fig. 4). Displacements and rotations at nodal points

\begin{tabular}{cccccccccc}
\hline \hline$u_{b}^{A}$ & $u_{c}^{A}$ & $\varphi^{A}$ & $u_{a}^{B}$ & $u_{b}^{B}=u_{c}^{B}$ & $\varphi^{B}$ & $u_{a}^{C}$ & $u_{b}^{C}$ & $u_{c}^{C}$ & $\varphi^{C}$ \\
\hline-0.064 & -0.151 & -0.011 & -0.065 & -0.065 & 0 & -0.131 & -0.067 & 0.021 & 0.012 \\
\hline
\end{tabular}

Table 4 Continuous beam over two spans (Fig. 4). Internal forces at nodes $A$ and $B$

\begin{tabular}{cccccc}
\hline \hline Node $A$ & Node $B$ & & & & \\
$\mathcal{Q}_{e 1}(0)$ & $\mathcal{N}_{e 2}^{a}(0)$ & $\mathcal{N}_{e 2}^{b}(0)$ & $\mathcal{N}_{e 2}^{c}(0)$ & $\mathcal{Q}_{e 2}(0)$ & $\mathcal{M}_{e 2}(0)$ \\
\hline $9.53 \mathrm{kN}$ & $-5.38 \mathrm{kN}$ & $5.39 \mathrm{kN}$ & $-0.01 \mathrm{kN}$ & $15.47 \mathrm{kN}$ & $-743.04 \mathrm{kNcm}$ \\
\hline
\end{tabular}

The values of the nodal displacements and rotations are presented in Table 3 . The displacements are given in centimeters and the rotations in radians.

The axial and shear forces and the bending moment in layers $a, b, c$ at nodal points are shown in Table 4. Only the non-zero values are shown.

Fig. 5 shows the variations of $\Delta^{a b}, \Delta^{b c}, \mathcal{N}, \mathcal{Q}, \mathcal{M}$ along the axis of each layer. It can be seen that the interlayer slips reach their maxima at the end supports. Graphs of slips $\Delta^{a b}$ and $\Delta^{b c}$ nearly agree. The lower $(a)$ and the upper layer $(c)$ are subjected to a considerable axial force in contrast to the intermediate layer $(b)$, the axial force of which is negligible. The resultant axial force of the composite beam is, obviously, equal to zero $\left(\mathcal{N}=\mathcal{N}^{a}+\mathcal{N}^{b}+\mathcal{N}^{c}=0\right)$. Note that when $\mathcal{N}^{a}$ is tensile, $\mathcal{N}^{c}$ is compressive, and vice versa. In contrast to axial forces in layers, the signs of the shear forces and bending moments are equal an all ayers. Their values are only roughly proportional to the bending rigidity of the layer. The largest portion of the shear force and the bending moment is taken over by the lower layer $a$, while the contribution of layer $b$ to the shear and bending capacity of the beam is rather small.

The influence of the different interlayer slip moduli on the values of various static and kinematic quantities is also examined. Fig. 6 shows the graphs of $\Delta^{a b}, w, p_{n}^{b c}$ and $\mathcal{M}^{c}$ as a function of the interlayer slip moduli $K=K^{a b}=K^{b c}$. From Fig. 6 it is obvious that the interlayer slip modulus has an important influence on static and kinematic quantities. The slip $\Delta^{a b}$ between the layers $a$ and $b$ 

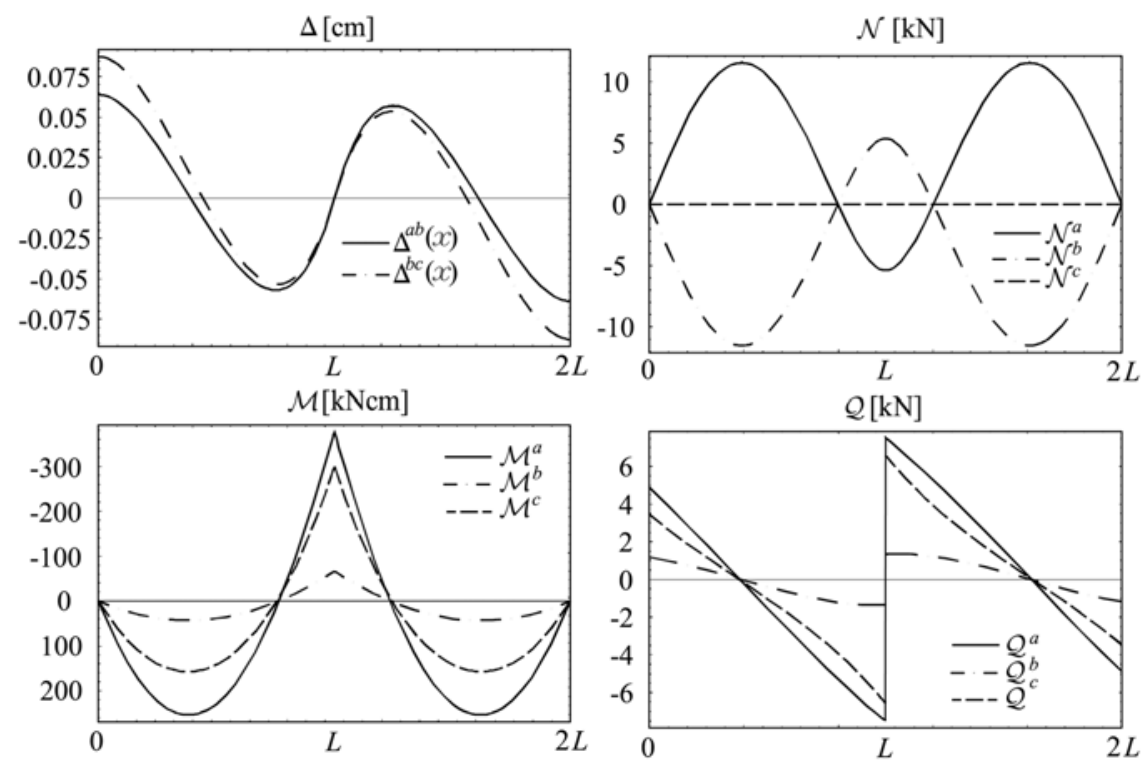

Fig. 5 Distribution of $\Delta^{a b}, \Delta^{b c}$ and $\mathcal{N}, \mathcal{Q}, \mathcal{M}$ in layers along the span. Continuous three-layer beam over two spans (Fig. 4).
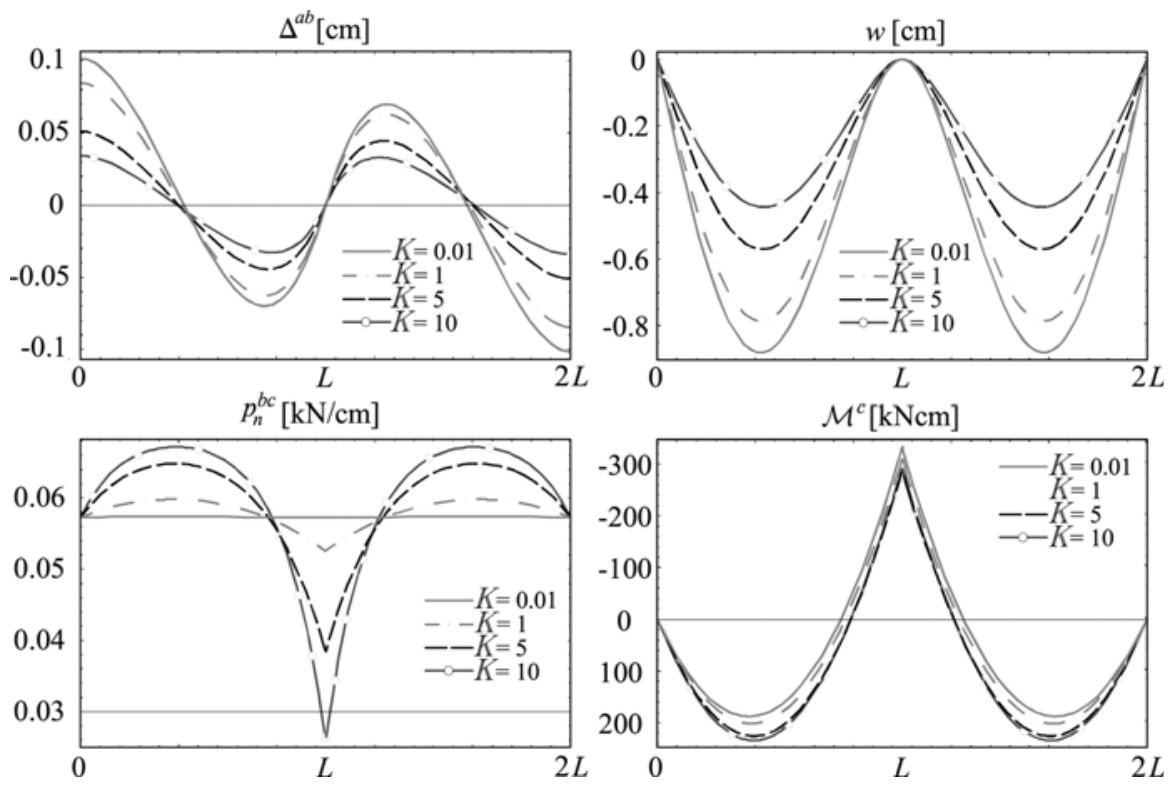

Fig. 6 Distribution of $\Delta^{a b}, w, p_{\eta}^{b c}$, and $\mathcal{M}^{c}$ along the span as a function of different values of the interlayer slip moduli, $K=K^{a b}=K^{b c}$

and the vertical deflection $w$ are much smaller for higher slip moduli $K$. Static quantities, such as interlayer stresses $p_{n}^{b c}$ between layers $b$ and $c$, and bending moment $\mathcal{M}^{c}$ in layer $c$ are, in contrast, higher for higher slip moduli. 


\section{Conclusions}

We proposed a mathematical model and found its analytical solution for the analysis of geometrically and materially linear layered, simply supported and continuous beams with an arbitrary number of layers and with variable material and geometric characteristics of the layers. The model is compared to the analytical model of Goodman and Popov (1968) for a simply supported three-layer beam, who assumed that the layers are identical. The model was applied to a two-span continuous composite timber beam. The advantage of the present exact analytical model is that, unlike in Goodman and Popov (1968), the thickness as well as material of the individual layers are arbitrary. The method is also suitable for obtaining referential or bench-mark solutions applicable in assessing the accuracy and convergence of newly developed finite element formulations.

\section{Acknowledgements}

The work of S. Schnabl was financially supported by the Ministry of Education, Science and Sport of the Republic of Slovenia under contract 3311-02-831625. Their support is gratefully acknowledged.

\section{References}

Adekola, U.O. (1968), "Partial interaction between elastically connected elements of a composite beam", Int. J. Solids Struct., 4, 1125-1135.

Ayoub, A. and Filippou, F.C. (2000), "Mixed formulation of nonlinear steel-concrete composite beam element", J. Struct. Eng., ASCE, 126(3), 371-381.

Ayoub, A. (2001), "A two-field mixed variational principle for partially connected composite beams", Finite Elements in Analysis and Design, 37, 929-959.

Čas, B. (2004a), "Non-linear analysis of composite beams with interlayer slip", PhD Thesis (in Slovenian), University of Ljubljana, Faculty of Civil and Geodetic Engineering.

Čas, B., Saje, M. and Planinc, I. (2004b), "Non-linear finite element analysis of composite planar frames with an interlayer slip", Comput. Struct., 82, 1901-1912.

Cosenza, E. and Pecce, M. (2001), "Shear and normal stresses interaction in coupled structural systems", $J$. Struct. Eng., ASCE, 127(1), 84-88.

Dall'Asta, A. and Zona, A. (2002), "Nonlinear analysis of composite beams by displacement approach", Comput. Struct., 80, 2217-2228.

Eurocode 5 (1993), "Design of timber structures, Part 1-1: General rules and rules for buildings", ENV 1995-1-1.

Fabbrocino, G., Manfredi, G. and Cosenza, E. (2000), "Analysis of continuous composite beams in cluding partial interaction and bond", J. Struct. Eng., ASCE, 126(11), 1288-1294.

Fabbrocino, G., Manfredi, G. and Cosenza, E. (2002), "Modelling of continuous steel-concrete composite beams: Computational aspects", Comput. Struct., 80, 2241-2251.

Faella, C., Martinelli, E. and Nigro, E. (2002), "Steel and concrete composite beams with flexible shear connection: 'exact' analytical expression of the stiffness matrix and applications", Comput. Struct., 80, 10011009.

Faella, C., Martinelli, E. and Nigro, E. (2003), "Shear connection nonlinearity and deflections of steel-concrete composite beams with: A simplified method", J. Struct. Eng., ASCE, 129(1), 12-20.

Fragiacomo, M., Amadio, C. and Macorini, L. (2004), "Finite-element model for collapse and long-term analysis 
of steel-concrete composite beams", J. Struct. Eng., ASCE, 130(3), 489-497.

Gatessco, N. (1999), "Analytical modeling of nonlinear behavior of composite beams with deformable connection", J. Const. Steel Res., 52, 195-218.

Girhammar, U.A. and Gopu, V.K.A. (1993), "Composite beam-columns with interlayer slip-exact analysis", $J$. Struct. Eng., ASCE, 199(4), 1265-1282.

Girhammar, U.A. and Pan, D. (1993), "Dynamic analysis of composite member with interlayer slip", Int. J. Solids Struct., 30(6), 797-823.

Goodman, J.R. and Popov, E.P. (1968), "Layered beam systems with interlayer slip", J. Struct. Div., ASCE, 94(11), 2535-2547.

Goodman, J.R. and Popov, E.P. (1969), "Layered wood systems with interlayer slip", Wood Science, 1(3), 148158.

Heinisuo, M. (1988), “An exact finite element technique for layered beams", Comput. Struct., 30(3), 615-622.

Jasim, N.A. (1997), "Computation of deflections for continuous composite beams with partial interaction", Proc. of The Institution of Civil Engineers, Structures and Buildings, 122, 347-354.

Jasim, N.A. and Mohamad, A.A. (1997), "Deflections of composite beams with partial shear connection", The Struct. Eng., 75(4), 58-61.

Jasim, N.A. (1999), "Deflections of partially composite beams with linear connector density", J. Const. Steel Res., 49, 241-254.

Kristek, V. and Studnicka, J. (1982), "Analysis of composite girders with deformable connectors", Proc. of The Institution of Civil Engineers, Part 2, 73, 699-712.

Linden, M.V. (1999), “Timber-concrete composite beams", HERON, 44(3), 215-239.

McCutheon, W.J. (1986), "Stiffness of framing members with partial composite action", J. Struct. Eng., ASCE, 112(7), 1623-1637.

Newmark, N.M., Siess, C.P. and Viest, I.M. (1951), "Test and analysis of composite beams with incomplete interaction", Proc. of the Society for Experimental Stress Analysis, 1, 75-92.

Nie, J., Fan, J. and Cai, C.S. (2004), "Stiffness and deflection of steel-concrete composite beams under negative bending", J. Struct. Eng., ASCE, 130(11), 1842-1851.

Oven, V.A., Burgess, I.W., Plank, R.J. and Abdul Wali, A.A. (1997), "An analytical model for the analysis of composite beams with partial interaction", Comput. Struct., 62(3), 493-504.

Plum, D.R. and Horne, M.R. (1975), "The analysis of continuous composite beams with partial interaction", Proc. of The Institution of Civil Engineers, Structures and Buildings, Part 2, 59, 625-643.

Ranzi, G., Bradford, M.A. and Uy, B. (2003), "A general method of analysis of composite beams with partial interaction", Steel and Composite Structures, 3(3), 169-184.

Rassam, H.Y. and Goodman, J.R. (1971), "Design of layered wood columns with interlayer slip", Wood Science, 3(3), 149-155.

Reissner, E. (1972), “On one-dimensional nite-strain beam theory: The plane problem”, J. of Appl. Mech. and Physics (ZAMP), 23, 795-804.

Silfwerbrand, J. (1997), "Stresses and strains in composite concrete beams subjected to differential shrinkage", ACI Struct. J., 94(4), 347-353.

Smith, S.T. and Teng, J.G. (2001), "Interfacial stresses in plated beams", Eng. Struct., 23, 857-871.

Thompson, E.G., Goodman, J.R. and Vanderbilt, M.D. (1975), "Finite element analysis of layered wood systems", J. Struct. Div., ASCE, 101(ST12), 2659-2672.

Wang, Y.C. (1998), "Deflection of steel-concrete composite beams with partial shear interaction", J. Struct. Eng., ASCE, 124(10), 1159-1165.

Wheat, D.L. and Calixto, J.M. (1994), "Nonlinear analysis of two-layered wood members with inter-layer slip", J. Struct. Eng., ASCE, 120(6), 1909-1929.

Wolfram, S. (2003), Mathematica, Addison-Wesley Publishing Company. 